\title{
Le transfert d'information, moteur de l'histoire boursière américaine
}

\section{Michel Van der Yeught}

\section{(2) OpenEdition}

1 Journals

\section{Édition électronique}

URL : http://journals.openedition.org/asp/1271

DOI : 10.4000/asp.1271

ISBN : 978-2-8218-0392-3

ISSN : 2108-6354

\section{Éditeur}

Groupe d'étude et de recherche en anglais de spécialité

Édition imprimée

Date de publication : 1 mai 2003

Pagination : 3-19

ISSN : 1246-8185

Référence électronique

Michel Van der Yeught, « Le transfert d'information, moteur de l'histoire boursière américaine », ASp [En ligne], 39-40 | 2003, mis en ligne le 04 mai 2010, consulté le 30 avril 2019. URL : http:// journals.openedition.org/asp/1271 ; DOI : 10.4000/asp.1271

Ce document a été généré automatiquement le 30 avril 2019.

Tous droits réservés 


\title{
Le transfert d'information, moteur de l'histoire boursière américaine
}

\author{
Michel Van der Yeught
}

\section{Introduction}

1 Wall Street représente un phénomène si typiquement américain qu'il est difficile de ne pas y voir un produit spécifique de l'histoire, de la société et de la culture des États-Unis. Or, une des facettes de Wall Street concerne ses rapports avec le grand public américain et notamment les informations qui circulent entre ces deux mondes. Depuis ses origines, l'histoire de Wall Street est en effet marquée par le problème de l'information. Ceux qui disposent d'une information de qualité, avant les autres, peuvent faire fortune grâce à la bourse. Les moins bien informés risquent de se ruiner. La lutte pour obtenir des renseignements est donc constante et le transfert d'information est un enjeu stratégique pour les opérateurs de Wall Street.

2 Dans cette perspective, il est intéressant d'étudier comment le problème du transfert d'information a façonné les marchés boursiers américains au cours de leurs deux cents ans d'existence et comment il a influencé leurs relations avec le grand public. Le titre de cet article suggère même que le transfert d'information est d'une importance telle qu'il a joué un rôle dynamique et constructif de premier plan dans l'évolution de Wall Street.

Trois considérations motivent ce travail.

4 D'abord, l'actualité économique encourage à s'interroger sur le rôle de l'information dans la finance moderne. Les nombreux scandales qui secouent depuis deux ans les plus grandes entreprises d'Amérique et d'Europe montrent combien le transfert d'information entre les différents acteurs économiques constitue la base de la confiance générale indispensable au système économique libéral. Il n'est peut-être pas inutile dans ce contexte, de tenter de relire l'évolution de Wall Street dans cette perspective. 
5 Ensuite, les avancées de la recherche ont dégagé récemment une problématique d'analyse qui semble extrêmement fertile pour comprendre le phénomène de Wall Street. Les trois prix Nobel d'économie 2001, Akerlof, Stiglitz et Spence ont placé l'asymétrie d'information au cœur de leurs modèles explicatifs. L'analyse aura recours à cette problématique car la rationalité des acteurs boursiers paraît extrêmement dépendante de leur situation, favorable ou défavorable, par rapport à l'information.

Enfin, au stade actuel de ces travaux, il semble que peu de chercheurs aient déjà tenté de lire l'histoire boursière américaine de ce point de vue particulier ${ }^{1}$. D'une part, les économistes utilisent l'asymétrie d'information pour construire des modèles mathématiques destinés à expliquer le fonctionnement du marché des véhicules d'occasion (Akerlof), le marché de l'emploi (Spence) ou le marché des assurances (Stiglitz) (Nobel Prize Lectures 2001). D'autre part, les grands historiens de Wall Street (Sobel, Michie, Geisst, Gordon) évoquent à chaque page de leurs travaux l'asymétrie d'information inhérente aux marchés boursiers sans jamais utiliser le concept lui-même et sans en faire un objet d'étude spécifique.

7 Dans ces conditions, il a paru utile de tenter de défricher à l'aide de nouveaux instruments ce terrain historique déjà bien connu. L'objectif est ici de mieux le comprendre grâce à un angle de vue inédit et de replacer la crise actuelle de l'information financière dans la continuité de l'histoire de Wall Street.

8 Cela dit, les dettes de ce travail sont nombreuses. Outre la problématique de l'asymétrie d'information, il s'inspire des travaux de deux historiens de l'économie: Douglass C. North (Prix Nobel d'économie 1993) et Charles Kindleberger². Il repose également sur la littérature boursière américaine (histoires, témoignages, chroniques, ouvrages techniques) et sur un passage de la Théorie Générale de Keynes.

La démarche suivie est la suivante.

9 Afin d'analyser deux siècles d'histoire boursière à partir du transfert d'information, il apparaît nécessaire de construire un modèle explicatif et une grille de lecture adaptés. À défaut, on s'expose à expliquer des périodes différentes à partir de concepts différents et par suite à ignorer les similitudes ou au contraire les évolutions qui sont les éléments de base d'une meilleure compréhension de l'histoire. Tout au contraire, un modèle explicatif préalablement construit précise les concepts et les relations opérationnelles qui existent entre eux et il met ainsi mieux en relief les mouvements ou les immobilismes de l'histoire. La première partie sera consacrée à une exposition rapide de ce modèle.

Dans une deuxième partie, ce modèle explicatif sera appliqué aux deux siècles de l'évolution boursière américaine. Il permettra de découper cette période d'une façon inédite, d'en faire une lecture renouvelée et d'y découvrir des événements ou des personnages négligés, soudain replacés au cœur du débat par cette problématique. Enfin il apportera peut-être un nouvel éclairage sur les scandales récents de type Enron où la question du transfert d'information est centrale.

\section{Présentation du modèle explicatif}

Le modèle explicatif repose sur trois piliers :

- Il précise le mécanisme de l'asymétrie d'information et il l'applique au domaine boursier, particulièrement dans le contexte américain. Il définit également les caractéristiques des acteurs qui interviennent dans ce mécanisme. 
- Il précise ensuite les différents types d'information boursière transférée de certains acteurs à d'autres, ce qui revient à dresser une typologie de ces informations.

- Enfin, il distingue les différents canaux par lesquels l'information peut circuler, ce qui revient à dresser une typologie des moyens de transférer l'information. différentes périodes étudiées.

\subsection{Asymétrie d'information et bourses américaines} dit, les professionnels, qui par métier maîtrisent les transactions boursières, se sont durablement installés du côté favorable de l'asymétrie d'information. En centralisant les renseignements venant des marchés et des investisseurs, ils sont devenus des insiders structurels bien placés pour en profiter les premiers. De ce point de vue, Wall Street représente la structuration socio-professionnelle de l'asymétrie d'information boursière ${ }^{4}$, c'est-à-dire du privilège d'information, par rapport au reste de la population américaine. Cette constante n'empêche pas la concurrence de se développer entre les insiders de Wall Street qui sont loin de constituer un monde homogène. Parmi eux, en effet, l'asymétrie 
d'information existe aussi et suscite à l'infini des groupes instables d'insiders et d'outsiders

17 Les outsiders, ou non-initiés, sont ceux qui se trouvent du côté défavorable de l'asymétrie d'information. De façon similaire, leurs caractéristiques sont tout aussi instables que celles des insiders, c'est-à-dire qu'ils peuvent tout aussi soudainement passer à l'état d'initiés en fonction des informations qu'ils obtiennent. Cela dit, de la même manière que pour les insiders, l'évolution historique a durablement fixé dans ce groupe les investisseurs américains non-professionnels des marchés financiers, les membres des classes moyennes qui gèrent un portefeuille boursier, les petits porteurs individuels qui habitent les campagnes ou les petites villes. Depuis le début du XXe siècle, une expression familière en est venue à désigner les outsiders par rapport à Wall Street, c'est Main Street (Perkins 1999 : 248). De ce point de vue, une grande partie de l'opposition Wall Street/ Main Street fréquemment évoquée par la presse américaine est la cristallisation à l'échelle nationale de l'opposition insider/outsider non seulement du strict point de vue financier, mais également au niveau social et pour ainsi dire culturel. Ce type de cristallisation est sans équivalent dans le reste du monde et représente un des grands éléments constitutifs de la civilisation américaine.

L'opposition insider/outsider est aussi vieille que les marchés boursiers eux-mêmes. En anglais, insider signifie celui qui est à l'intérieur. Et de fait les premiers initiés en titre de l'histoire boursière américaine furent les courtiers signataires du Buttonwood Agreement de 1792 qui constitue l'acte fondateur de la Bourse de New York (Sobel 1970 : 12-13). Les 24 membres de ce club privé quittèrent la rue où tous les courtiers opéraient jusqu'alors pour se réunir à l'intérieur de cafés durant la tenue de leurs sessions. Les autres, ceux qui ne furent pas admis, restèrent dans la rue et continuèrent à opérer dehors sur le pavé (Gordon 1999: 39-40). Ces exclus représentent les premiers outsiders de l'histoire boursière américaine, et cette scission de la communauté des courtiers en insiders et outsiders institutionalisa pour la première fois l'asymétrie d'information boursière dès la fin du XVIII ${ }^{e}$ siècle. Depuis ses origines jusqu'aux malversations d'Enron en passant par les grands spéculateurs $\mathrm{du} \mathrm{XIX}^{\mathrm{e}}$ siècle, la tradition boursière américaine est essentiellement faite d'histoires spectaculaires et croustillantes où les initiés bien informés de Wall Street ruinent les petits investisseurs de Main Street.

\subsection{Typologie de l'information boursière}

19 L'information boursière n'a de valeur que si elle est sensible, c'est-à-dire susceptible d'affecter le cours des valeurs. La tradition boursière distingue deux types différents d'information. Elle oppose les opérations boursières spéculatives (qui visent des gains à court terme réalisés en tirant parti des fluctuations des cours) des opérations d'investissement (qui visent des gains à long terme grâce à la valorisation progressive des actifs d'une société). On peut en déduire aisément qu'il existe deux grandes catégories d'informations. Celle qui alimente la spéculation (qu'on appellera information spéculative) et celle qui contribue à l'investissement (qu'on appellera information d'investissement).

Cette typologie à deux termes est claire et pratique, mais elle semble incomplète. Il existe en effet un autre type d'information que Keynes met bien en évidence (Keynes 1936: chapitre 12). Il distingue classiquement entre la spéculation (c'est la célèbre métaphore du concours de beauté) et l'investissement. Mais il ajoute à l'analyse un élément 
important. Il explique que l'ensemble des activités boursières repose sur une véritable "convention" qui représente l'avis global de la communauté économique sur l'évaluation actuelle du marché en fonction des informations dont elle dispose. Cette convention évolue avec le flux des informations mais tant qu'elle perdure, elle assure au marché sa continuité et sa stabilité.

La typologie proposée adopte cette analyse et il en découle qu'il existe un troisième type d'information boursière qui n'est ni purement spéculative, ni d'investissement. C'est une information propre à alimenter la convention générale de la communauté à l'égard du marché. Certes, cette information est évolutive et composite, mais elle contribue à faire perdurer la convention et elle assure au marché sa pérennité.

La typologie comprend donc les trois termes suivants :

- Est appelée information de type 1 l'information spéculative. Elle permet aux spéculateurs de deviner avant les autres l'état futur du marché, généralement à court terme, indépendamment du rendement escompté d'un investissement donné.

- Est appelée information de type 2 l'information conventionnelle (au sens keynésien de « convention » dans le chapitre 12 de la Théorie Générale). Elle alimente la communauté économique en renseignements sur l'évaluation actuelle du marché : actualité des entreprises, statistiques nationales, différents taux d'intérêt, cours des monnaies, indices boursiers et indicateurs économiques, rapports des agences de notation financière, etc.

- Est appelée information de type 3 les informations qui alimentent l'investissement à long terme dans les titres d'une société donnée : résultats financiers et comptables, données de base concernant l'entreprise, rapports d'audit, analyse financière approfondie, etc.

Les différents types d'information boursière étant posés, il importe à présent d'analyser les moyens par lesquels ils peuvent être transférés.

\subsection{Typologie des moyens de transfert de l'information boursière}

L'asymétrie d'information suscite une tension entre les insiders et les outsiders, ces derniers faisant pression sur les premiers pour obtenir les informations dont dépendent leurs plus-values. Ainsi se pose le problème de son transfert comme facteur de réduction de l'asymétrie d'information.

Les canaux par lesquels l'information peut circuler sont si nombreux qu'il apparaît nécessaire de leur donner une appellation commune. Plutôt que d'opter pour un terme général comme canal, vecteur ou medium, je propose de les appeler " éléments-janus » en référence au dieu Janus des Romains. Le dieu Janus était en effet doté de deux têtes opposées et le latiniste Pierre Grimal (Grimal 1945 : 104) ainsi que l'anthropologue James Frazer (Frazer 1922: 218-219) s'accordent pour voir en lui le dieu des portes, des arches et des passages. Je suis attaché à l'image du Janus car ses deux visages font symétriquement face aux initiés comme aux non-initiés, à Wall Street comme à Main Street, et ils facilitent la transmission d'information entre eux. En outre, ses deux bouches excluent les transferts à sens unique et suggèrent plutôt que l'information puisse circuler aussi bien dans un sens que dans l'autre. Dans le contexte de l'histoire boursière américaine, je définis donc les éléments-janus comme les moyens de transmission de l'information qui contribuent à réduire l'asymétrie d'information entre les initiés et les non-initiés, entre Wall Street et Main Street. janus technologiques, janus organisationnels et janus institutionnels. Dans cette 
typologie, je m'inspire des travaux de Douglass North, et notamment j'adopte sa distinction entre organisations et institutions (North 1994 : 1-2).

1. Les janus informels : il s'agit de vecteurs d'information interpersonnels comme les conversations privées, les échanges de lettres, les coups de téléphones et les courriers électroniques, les rumeurs, les conseils, les tuyaux boursiers données intuitu personnae, l'observation et l'espionnage. Les janus informels sont particulièrement bien adaptés au transfert d'information événementielle de type 1 (spéculatif).

2. Les janus technologiques proviennent du progrès technique : le télégraphe (1844), les câbles transatlantiques posés entre la Grande-Bretagne et les États-Unis (1867), le télescripteur (stockticker, 1867), le téléphone (1876), l'Internet. Ils transmettent l'information boursière de personne à personne mais aussi des cercles informés au grand public avec un fort potentiel multiplicateur. Ils réduisent, ou ont contribué à réduire fortement, les causes spatio-temporelles de l'asymétrie d'information. Historiquement, ils se sont développés à la fin du XIX ${ }^{\mathrm{e}}$ siècle, mis à part l'Internet qui représente actuellement la forme la plus évoluée des janus technologiques. Ils sont particulièrement aptes à transférer des informations de types 1 et 2.

Les éléments janus organisationnels et institutionnels : Ces deux dernières classes comportent des organisations et des institutions (au sens de Douglass North) qui se donnent pour mission explicite de réduire l'asymétrie d'information boursière entre les cercles financiers bien informés et le grand public des investisseurs. Ils représentent par là même les janus médiateurs par excellence. Placés à égale distance de Wall Street et de Main Street, ils défendent équitablement les intérêts des deux parties.

29 3. Les janus organisationnels regroupent les organes de la presse financière et économique (Wall Street Journal, Dow Jones et Barron's, BusinessWeek), les médias audiovisuels pourvoyeurs d'information sur les marchés (Reuters, Bloomberg, CNBC), les agences spécialisées d'information et de notation financière (Dow Jones Averages, Standard \& Poor's, Moody's), les cabinets de comptabilité et d'audit (les Big Four).

4. Les janus institutionnels sont représentées par les autorités responsables de la qualité $\mathrm{du}$ transfert d'information entre initiés et non-initiés. Aux États-Unis, le janus institutionnel le plus important est la Securities and Exchange Commission (la SEC) fondée en 1934 à la suite du Grand Krach de 1929. La SEC s'appuie sur d'autres vecteurs de transfert d'information qui sont moins connus mais qui méritent également de figurer parmi les janus institutionnels. Les normes comptables GAAP (Generally Accepted Accounting Principles) en vigueur aux États-Unis ainsi que les blue sky laws, c'est-à-dire les exigences légales d'information boursière imposées par les différents États américains, font également partie des janus institutionnels de ce pays. Il va sans dire que leur efficacité dépend de leur indépendance stricte par rapport aux deux pôles d'influence que représentent Wall Street et Main Street. Le double visage équilibré de l'élément-janus prend ici toute son importance. En effet, si l'un des visages prend l'ascendant sur l'autre, par exemple si la SEC fait le jeu de Wall Street ou se révèle incapable de défendre les intérêts de Main Street, l'asymétrie d'information s'accentuera au lieu de se réduire et une crise de confiance se développera.

31 Les janus organisationnels et institutionnels sont aptes à transmettre tous les types d'information mais leur mission première est d'assurer la diffusion d'informations de types 2 et 3 destinées à construire une convention stable et continue sur l'état du marché et à informer et à éduquer durablement le grand public américain. 
Le modèle explicatif est désormais posé et ses différents composants figurent dans le tableau 1.

Tableau 1. Modèle explicatif

\begin{tabular}{|l|l|}
\hline \multicolumn{2}{|c|}{ Modèle explicatif : éléments de la grille de lecture } \\
\hline $\begin{array}{l}\text { Typologie des } \\
\text { informations }\end{array}$ & Spéculative (visant des gains à court terme sur fluctuations rapides) \\
\hline Type 1 & $\begin{array}{l}\text { Conventionnelle (visant à constituer une opinion publique du } \\
\text { marché) }\end{array}$ \\
\hline Type 2 & $\begin{array}{l}\text { Investissement (information aidant à investir à long terme dans } \\
\text { certaines sociétés) }\end{array}$ \\
\hline Type 3 & $\begin{array}{l}\text { Conversations, lettres, courriels, rumeurs, conseils, tuyaux } \\
\text { boursiers, espionnage }\end{array}$ \\
\hline Typologie des janus & Télégraphe, téléphone, ticker, câble, Internet \\
\hline Informels & $\begin{array}{l}\text { Presse financière, indices, agences d'information financière, firmes } \\
\text { comptables et d'audit }\end{array}$ \\
\hline Technologiques & Securities and Exchange Commission, blue sky laws, GAAP \\
\hline Organisationnels & Institutionnels
\end{tabular}

\section{Application du modèle d'explication à l'histoire boursière américaine}

33 Les historiens de Wall Street gardent généralement une lecture événementielle de l'histoire et ils découpent les périodes en fonction des grands événements qui influencent les marchés (guerre d'Indépendance, guerre de Sécession, crise de 1929, etc.). L'analyse proposée ici se démarque quelque peu de leur approche. En effet, relire l'histoire boursière américaine du point de vue de l'asymétrie d'information conduit à la découper prioritairement à partir de cette problématique et en fonction de sa logique propre. C'est ainsi qu'il est possible de distinguer trois grandes périodes structurellement façonnées par certains types de transferts d'information : des années 1790 aux années 1850, des années 1860 aux années 1910 et des années 1920 à nos jours.

\subsection{Première Période : des années 1790 aux années 1850}

34 Au cours de cette période, le monde boursier américain se met progressivement en place et se développe au rythme du pays, d'abord lentement (1790-1820), puis de plus en plus rapidement (1820-1850). Les marchés boursiers (Philadelphie 1790 et New York 1792, 
rejoints par Boston en 1834) sont embryonnaires et fonctionnent comme des clubs privés et exclusifs d'insiders dont la fonction première est de protéger les intérêts de leurs membres. Ils pratiquent une politique malthusienne et rejettent fréquemment les postulants. Les outsiders comprennent les exclus des clubs boursiers et une petite partie de la population locale qui se laisse tenter par la spéculation.

L'asymétrie d'information est pratiquement absolue. Les insiders qui assistent aux séances de cotation constituent la source quasi exclusive de l'information sur les cours. Les outsiders en sont réduits à récupérer des miettes de renseignements grâce aux tuyaux et aux rumeurs que laissent échapper les initiés et les grands opérateurs de l'époque. L'activité boursière est essentiellement spéculative (banques, compagnies d'assurance, canaux, chemins de fer) et l'évolution des marchés résulte de batailles sans merci livrées entre des cliques de haussiers appelés bulls et de baissiers appelés bears. Ils vendent à découvert (short sales) et organisent des corners pour faire monter les cours et ruiner leurs adversaires ${ }^{5}$.

L'information recherchée est essentiellement de type 1 (qui achète quoi, quel coup de bourse se prépare, qui s'associe avec qui, quels titres montent, quels titres descendent?). Les éléments-janus sont informels (tuyaux boursiers, espionnage, conversations, conseils, courriers). Les informations de types 2 et 3 sont embryonnaires: quelques renseignements sur la clôtures des cours dans certaines gazettes, quelques tableaux comparatifs. La comptabilité reste élémentaire (bookkeeping) et est quasiment laissée à la discrétion des professionnels de chaque entreprise. Les janus technologiques n'existent pas (les liaisons télégraphiques transcontinentales se développent seulement dans les années 1850) et les janus organisationnels sont inexistants ou embryonnaires. La presse financière en est à ses balbutiements et la presse en général est souvent vénale et sensationnaliste. Que ce soit au niveau des différents États ou au niveau fédéral, aucune réglementation n'encadre l'activité d'investissement. Par conséquent, les janus institutionnels sont également absents de la période.

Les insiders verrouillent ou manipulent les transferts d'information. L'origine de la bourse de New York elle-même en 1792 vient d'une manœuvre d'insiders (Wachtel 1996). Les sessions du New York Stock Exchange (NYSE) sont strictement privées et tenues secrètes. Les membres ont interdiction de dévoiler la nature des transactions et les prix pratiqués. Ils ne peuvent pas recevoir des messages pendant les séances de cotation.

Les outsiders espionnent les insiders en les observant pendant les séances (Garvy 1944 : 128-143, 129 note 9), en louant des salles adjacentes à la salle des séances du NYSE et en perçant le mur pour écouter les cotations (Sobel 1970 : 17), en regardant par le trou des serrures (Garvy : 130 note 11). Une asymétrie d'information se développe également entre bourses rivales. Philadelphie ne reçoit les navires et les nouvelles venant d'Europe qu'après New York qui se trouve ainsi du côté favorable de l'asymétrie. Pour rétablir l'équilibre, les membres de la bourse de Philadelphie mettent en service un réseau de signaux lumineux codés qui transmettent l'information de New York à Philadelphie en 10 minutes environ. Le système resta opérationnel jusqu'à l'installation du télégraphe en 1846. C'est le seul janus technologique qu'on enregistre à l'époque (Philadelphia Stock Exchange : 1). Le tableau 2 récapitule les grandes caractéristiques de la période en terme de transferts d'information. 
Tableau 2. Transferts d'information : Années 1790 - 1850

\begin{tabular}{|l|l|l|}
\hline \multicolumn{1}{|c|}{ Éléments d'analyse } & Commentaires & \multicolumn{1}{|c|}{ Exemples } \\
\hline $\begin{array}{l}\text { Asymétrie } \\
\text { d'information }\end{array}$ & Quasi absolue & Les insiders dominent la bourse \\
\hline Types d'information & & \\
\hline Type 1 & Prédominante & Coups boursiers, luttes bulls/bears \\
\hline Type 2 & Rare et limitée & Cours de clôture, quelques tableaux comparatifs \\
\hline Type 3 & Embryonnaire & Comptabilité primitive \\
\hline Types d'éléments- \\
janus & information de type 1 et janus informels prédominants \\
\hline Informels & Prédominants & $\begin{array}{l}\text { Courriers, tuyaux, conversations, rumeurs, } \\
\text { espionnage }\end{array}$ \\
\hline Technologiques & Inexistants & (sauf sémaphore New York-Philadelphie) \\
\hline Organisationnels & Embryonnaires & $\begin{array}{l}\text { Quelques gazettes commerciales, presse vénale ou } \\
\text { sensationnaliste }\end{array}$ \\
\hline Institutionnels & Inexistants & générale : asymétrie d'information quasi absolue \\
\hline
\end{tabular}

\subsection{Deuxième Période : des années 1860 aux années 1910} d'information forte, activité et information spéculatives) mais également par une évolution profonde et structurelle des janus et des informations transmises. Ces évolutions transforment radicalement l'activité boursière particulièrement au cours des années 1860 qui constitue une décennie de rupture avec la période précédente.

À partir des années 1850-60, de multiples ruées vers l'or poussent un nombre croissant de pionniers vers l'Ouest et entretiennent une ambiance de fièvre spéculative. Washington finance la guerre de Sécession par des bons du Trésor massivement souscrits qui sensibilisent des millions d'Américains à l'investissement financier (Geisst 1997 : 53-58). Le nombre des bourses augmente à une vitesse incroyablement rapide : il passe de trois dans les années 1830 à plus de 200 dans les années 1860 (Michie 1987 : 167). La plupart sont éphémères et servent de véhicules à la spéculation sur les mines, les chemins de fer, les compagnies pétrolières, mais leur multiplication témoigne d'un engouement certain des Américains pour l'investissement et la spéculation. 
41 La période est marquée par une multiplication rapide des janus technologiques que le progrès technique met à la disposition d'investisseurs chaque année plus nombreux : télégraphe (1844), câble transatlantique Londres-New York (1867) (Michie 1987 : 187-189), télescripteur (1867), téléphone (1876) (Michie: 172-176). Ces janus transmettent informations et ordres aux quatre coins des États-Unis et de part de d'autre de l'Atlantique. Un chercheur de l'université de Constance en Allemagne a montré que le développement rapide du télescripteur (stockticker) a révolutionné le fonctionnement des mécanismes boursiers en codifiant l'information boursière, en la dépersonnalisant et en la transformant en flux continu (Preda 2002). Même si ces transferts servent généralement des fins spéculatives, ils offrent une base fiable et permanente à la constitution des cours de bourse. Au cours de cette période, les janus technologiques mettent à la disposition du grand public des informations naguère réservées aux insiders et ils réduisent donc considérablement l'asymétrie d'information.

Le volume d'activité en forte croissance ainsi que ces transferts d'information massifs et continus, obligent les insiders de Wall Street à faire évoluer leurs pratiques. Les enchères à tour de rôle (call auctions) laissent progressivement la place aux enchères en continu ( continuous auctions) animées sur le parquet par des spécialistes à partir de 1871 (Michie 1987 : 184-185. Colbert 2001: 52). Actuellement, la bourse de New York fonctionne toujours selon ce mécanisme mis en place dans les années 1870 et les transferts d'information apparaissent comme un des moteurs essentiels de l'évolution boursière aux États-Unis.

Les insiders de cette période sont partagés sur l'intrusion de ces janus technologiques dans le jeu boursier. Dans un premier temps (années 1860), ils y voient un danger pour leur monopole sur l'information de type 1 qui restait le privilège de Wall Street. Par conséquent, ils s'efforcent d'entretenir l'asymétrie à leur avantage (Michie 1987 : 200). Dans un second temps (années 1870) Ils cessent progressivement leur combat contre l'usage libre des janus technologiques lorsqu'ils comprennent que la lutte est vaine et surtout quand ils se rendent compte que les transferts massifs d'information renforcent la position de New York au détriment des nombreuses bourses régionales et locales. C'est ainsi que tout en luttant pour restreindre l'usage libre des janus technologiques, les insiders les adoptent rapidement pour eux-mêmes (Angel 1998 : 10). Par exemple, le téléphone fut installé à la bourse de New York en 1878, deux ans à peine après son invention en 1876 (New York Stock Exchange : Significant Historical Dates).

Une révolution similaire touche les janus organisationnels que des hommes de conviction inventent et construisent au cours de cette période. Leurs travaux débutent dans les années 1840 et 1850 mais ne commencent à marquer le public que dans les années 1860 . Henry Varnum Poor (qui est à l'origine de l'agence d'information Standard \& Poor's) publie un journal (The American Railroad Journal) et un manuel des chemins de fer américains qui constituent deux mines de renseignements techniques et financiers précieux pour les investisseurs. Sa devise était « The investor's right to know », l'investisseur a le droit de savoir (Chandler 1981: 205-225). John Moody (fondateur de la firme de notation Moody's) fait de même pour les sociétés industrielles en 1900.

Un autre promoteur de l'information financière, Charles Dow, crée successivement trois janus organisationnels dont l'importance fut et reste considérable. Il fonde le Wall Street Journal (1884), il calcule la première moyenne boursière (l'indice Dow Jones, 1884 puis 1896) et il pose les bases de la théorie de Dow (the Dow Theory), première théorie boursière à base d'observations scientifiques. Ces trois janus existent toujours actuellement et 
Charles Dow peut être considéré comme un pionnier de premier plan dans le domaine des janus organisationnels (Hamilton 1922 : 21-29). Les mécanismes d'information qu'il a mis au point ont puissamment façonné les marchés boursiers américains. Il est à remarquer que ni Poor, ni Moody, ni Dow ne furent des insiders de Wall Street.

Ces janus organisationnels contribuèrent à transmettre de l'information de type 2 ainsi que des embryons d'information de type 3. Ils alimentèrent les investisseurs en données qui fondent la convention générale du public sur la valorisation du marché. Avant l'indice Dow Jones, seuls les insiders pouvaient se faire une idée approximative des évolutions du marché. Or, du jour au lendemain, l'indice permit à des millions d'Américains de consulter le pouls de la bourse de New York et d'en mesurer les fluctuations quotidiennes de façon simple et arithmétique. Le transfert d'information fut considérable et contribua au développement embryonnaire d'une opinion publique boursière.

$47 \mathrm{Au}$ cours de cette période les janus institutionnels sont encore rares. Il faut souligner cependant que la bourse de New York commence à exiger des sociétés qu'elle cote des informations financières minimales. Par ailleurs, à la suite d'auditions organisées en 1912 par le Congrès sur le monopole financier exercé par Wall Street, une certaine prise de conscience se développe, dans le grand public et chez certains représentants des autorités, qu'il revient à l'État fédéral de limiter les abus du monopole exercé par les insiders de Wall Street (Sobel 1970: 213-271). Le tableau 3 récapitule les grandes caractéristiques de cette période.

Tableau 3. Transferts d'information : Années 1860 - 1910

\begin{tabular}{|c|c|c|}
\hline Éléments d'analyse & Commentaires & Exemples \\
\hline $\begin{array}{l}\text { Asymétrie } \\
\text { d'information }\end{array}$ & $\begin{array}{l}\text { Toujours forte mais } \\
\text { décroissante }\end{array}$ & \\
\hline $\begin{array}{l}\text { Types } \\
\text { d'information }\end{array}$ & & \\
\hline Type 1 & Répandue & Tuyaux, rumeurs, \\
\hline Type 2 & $\begin{array}{l}\text { En développement } \\
\text { rapide }\end{array}$ & $\begin{array}{l}\text { Analyses financières, nouvelles économiques, } \\
\text { indicateurs finan-ciers, méthodes } \\
\text { d'investissement }\end{array}$ \\
\hline Type 3 & Embryonnaire & $\begin{array}{l}\text { Information d'investissement (Poor et } \\
\text { Moody), comptabilité primitive }\end{array}$ \\
\hline $\begin{array}{l}\text { Types d'éléments- } \\
\text { janus }\end{array}$ & & \\
\hline Informels & $\begin{array}{l}\text { Nombreux mais en } \\
\text { importance décroissante }\end{array}$ & Courriers, conversations, tuyaux \\
\hline Technologiques & $\begin{array}{l}\text { En développement très } \\
\text { rapide }\end{array}$ & Télégraphe, ticker, téléphone, câble \\
\hline
\end{tabular}




\begin{tabular}{|l|l|l|}
\hline Organisationnels & $\begin{array}{l}\text { En développement } \\
\text { soutenu }\end{array}$ & $\begin{array}{l}\text { Presse (Wall Street Journal), indice Dow Jones, } \\
\text { études de Poor et Moody, Dow Theory }\end{array}$ \\
\hline Institutionnels & Embryonnaires & $\begin{array}{l}\text { Exigences du NYSE en information et } \\
\text { auditions du Congrès }\end{array}$ \\
\hline \multicolumn{2}{|c|}{ Tendance générale : transferts massifs d'information de type 2 grâce aux janus } \\
organisationnels en développement rapide
\end{tabular}

\subsection{Troisième période : des années 1920 à nos jours}

48 Cette période est cruciale parce que pour la première fois dans l'histoire boursière américaine, le transfert d'information devient l'objet d'une prise de conscience publique et un enjeu national. Pour la première fois, les autorités américaines se donnent pour mission de réduire l'asymétrie d'information boursière et d'assurer la qualité de son transfert.

49 Cette période est marquée par la hausse spéculative des années vingt accompagnée de l'augmentation rapide des Américains qui jouent en bourse. On estime à $25 \%$ la proportion de la population qui possède des titres, taux encore jamais atteint à l'époque, ni aux États-Unis ni ailleurs. Aux investisseurs traditionnels des classes supérieures s'ajoutent de 1927 à 1929 un nombre considérable de boursicoteurs appartenant aux classes moyennes et populaires. En 1920, Sinclair Lewis publie un roman au succès foudroyant, Main Street, où se miroite l'Amérique des campagnes et des petites villes, particulièrement celles du Middle West. La facette financière de l'expression Main Street en vient à définir tous les Américains qui s'intéressent à la bourse sans appartenir à Wall Street.

50 L'opposition Wall Street/Main Street, occultée par le marché haussier des années 1920 dont tout le monde profite, s'exacerbe après le krach de 1929 et pendant la Grande Dépression. Le public est persuadé que Wall Street est responsable du désastre et, de fait, les auditions du Congrès animées par le juge Ferdinand Pecora (Pecora Hearings) révèlent les abus et les privilèges des grands opérateurs de Wall Street, tel « Jack» Morgan le fils du célèbre banquier John Pierpont Morgan (Pecora 1939: 4-40). En 1938, Main Street, révulsée, voit le scandale atteindre l'intouchable président de la bourse de New York, Richard Whitney, convaincu de fraude et emprisonné au pénitencier de Sing-Sing (Colbert 2001 : 150-154. Gordon 1999 : 243-248).

51 Ces événements dramatiques entraînent une réaction du gouvernement qui engage, sous l'impulsion de Roosevelt, une réglementation stricte des marchés financiers et une réforme complète du transfert d'information boursière. Pour la première fois, les autorités fédérales interviennent pour rééquilibrer les flux d'information entre initiés et non-initiés. En 1934, la SEC est instituée pour faire appliquer le Securities Act de 1933 et le Securities Exchange Act de 1934. C'est ainsi que se mettent en place des janus institutionnels fédéraux dont la mission essentielle est de lutter contre l'asymétrie d'information. Le transfert d'information joue donc à nouveau un rôle clé dans la modernisation de Wall Street.

La mission donnée à la SEC fut de limiter la diffusion d'information de type 1 et les avantages qui lui sont liés, et de promouvoir l'information de type 3 pour encourager 
l'investissement à long terme et redonner confiance à Main Street. Fidèle à la tradition libérale américaine, l'État n'entrave pas la cotation de sociétés spéculatives ou les investissements sans garanties. Il exige seulement que l'investisseur soit mis au courant des risques qu'il encourt grâce à la publication d'informations précises et détaillées. De même, la loi rend illégaux les profits provenant directement de l'asymétrie d'information : en 1934, l'insider trading (le délit d'initié) fut interdit dans toute l'Union.

Parallèlement, les sociétés émettrices de titres cotés en bourse doivent soumettre à la SEC une série de dossiers financiers et comptables extrêmement complets. Pour la première fois, ces informations de type 3 mises à la disposition du grand public sont standardisées au niveau fédéral et peuvent donc être comparées d'un État à l'autre. (Aux États-Unis, il n'existe pas de statut fédéral des sociétés ; chaque entreprise est enregistrée dans l'un des cinquante États de l'Union et doit respecter la législation de son État d'enregistrement.) La base de données EDGAR de la SEC actuellement disponible sur Internet (<sec.gov/ edgar>) est la dernière évolution du transfert d'information massif de type 3 exigé par les autorités fédérales dans les années 1930.

Comme il fallait s'y attendre, Wall Street, et notamment la vieille garde du NYSE, combattirent vigoureusement les réformes visant à réduire leurs privilèges informationnels (Geisst 1997 : 237-253). Son président, Richard Whitney, déclara en 1936 que le NYSE était « une institution parfaite » qui n'avait pas besoin de réformes. Wall Street n'accepta la nouvelle donne imposée par Washington que lorsque Whitney fut emprisonné. Les réformateurs de la bourse purent alors coopérer pleinement avec les autorités (Gordon 1999 : 248, 294).

Le découpage historique proposé ici opte pour un début de cette troisième période à partir des années 1920. En effet, les racines de toutes ces réformes précèdent les années trente d'une dizaine d'années environ. Avant que le gouvernement fédéral n'intervienne pour réglementer le transfert d'information boursière, une vingtaine d'États de l'Union avaient adopté des lois de même nature à partir des années 1910, les fameuses blue sky laws (Alvarez and Astarita 1995-2001). Ces blue sky laws, qui sont en fait des janus institutionnels dans notre typologie, inspirèrent de nombreuses dispositions reprises ensuite par la SEC. De même, les 35 bourses qui opéraient à l'époque avaient suivi l'exemple de New York bien avant les années 1930 et elles exigeaient des entreprises qu'elles cotaient des informations financières et comptables. Cependant, ces dispositions étaient peu appliquées et les bourses rivalisaient plutôt de laxisme pour attirer les entreprises (Davis 1999: 30-37). Néanmoins, les lois des années 1930 eurent plutôt pour effet de systématiser ce qui existait déjà sans être mis en œuvre, plutôt que de faire table rase du passé. Ce rappel montre en outre la relative inefficacité de mesures éparpillées dans les différents États ou dépendantes de l'auto-réglementation des marchés euxmêmes. La leçon de cette période est claire : seules les autorités fédérales furent capables d'incarner l'intérêt général et d'assurer des transferts d'information massifs de Wall Street vers Main Street. Le message ne doit pas être oublié aujourd'hui alors que de nombreux représentants de Wall Street estiment préférable de s'en remettre à l'autoréglementation ou aux simples lois du marché (Oesterle 2000).

Une deuxième justification de ce découpage historique tient à l'organisation de la profession comptable qui se mit en place dans les années 1910. Un comptable éminent du $\mathrm{XX}^{\mathrm{e}}$ siècle, Arthur Andersen, fonda son cabinet à Chicago en 1913 et contribua profondément au développement des techniques qui furent à l'origine des GAAP (Arthur Andersen \& Co 1963). Ces normes comptables, imposées par la SEC dans les années 1930, 
évoluèrent et parvinrent à maturité dans les années 1970. Pour ces raisons, les réformes lancées en 1934 trouvent leur origine dans une maturation profonde à partir des années 1910-20, maturation largement masquée par la décennie spéculative des années folles.

Une autre révolution du transfert d'information boursière marqua la période 1940-1950. Chose totalement nouvelle, l'un des insiders de Wall Street se donna pour priorité de lutter contre l'asymétrie d'information. Charles Merrill, l'un des deux fondateurs du réseau de courtage Merrill Lynch, s'opposa à l'establishment de Wall Street et prit parti pour les réformes proposées par les autorités fédérales (Perkins 1999: 127-143). Il se proposa en outre de redonner confiance à Main Street en jouant la carte de la transparence informationnelle. Il fit de sa firme un authentique janus organisationnel qui alimenta les petits investisseurs en informations de types 2 et 3 en qualité et en quantité totalement inédites à l'époque. Persuadé que l'avenir de l'industrie d'investissement passait par les petits porteurs, ce pionnier se fixa pour mission d'amener Wall Street à Main Street («Bring Wall Street to Main Street») en informant et en éduquant le public à des méthodes raisonnables et rentables d'investissement. La concurrence jouant son rôle, de nombreux opérateurs s'inspirèrent de ses méthodes et contribuèrent également à réduire l'asymétrie d'information entre Wall Street et Main Street.

Dans une très large mesure, Wall Street repose aujourd'hui sur des fondations construites dans les années 1930, fondations largement dépendantes du transfert d'information entre Wall Street et Main Street. Le tableau 4 récapitule les principales caractéristiques de cette période.

Tableau 4. Transferts d'information : Années 1920 à nos jours

\begin{tabular}{|l|l|l|}
\hline Éléments d'analyse & \multicolumn{1}{|c|}{ Commentaires } & \multicolumn{1}{|c|}{ Exemples } \\
\hline $\begin{array}{l}\text { Asymétrie } \\
\text { d'information }\end{array}$ & En net rééquilibrage & \\
\hline $\begin{array}{l}\text { Types } \\
\text { d'information }\end{array}$ & $\begin{array}{l}\text { Répandue mais concurrencée } \\
\text { d'information }\end{array}$ & Tuyaux, rumeurs, \\
\hline Type 1 & $\begin{array}{l}\text { En développement constant } \\
\text { Type 2 }\end{array}$ & $\begin{array}{l}\text { Analyses financières, nouvelles } \\
\text { économiques, indicateurs finan-ciers, } \\
\text { méthodes d'investissement }\end{array}$ \\
\hline Type 3 & $\begin{array}{l}\text { En développement rapide à } \\
\text { partir des années 1930 } \\
\text { 1920, 1990) }\end{array}$ & $\begin{array}{l}\text { Information financière officielle, normes } \\
\text { comptables }\end{array}$ \\
\hline $\begin{array}{l}\text { Types d'éléments- } \\
\text { janus }\end{array}$ & $\begin{array}{l}\text { Conversations, tuyaux, téléphone, } \\
\text { courriels, forums et « chat » d'Internet }\end{array}$ \\
\hline
\end{tabular}




\begin{tabular}{|l|l|l|}
\hline Technologiques & En développement rapide & ticker, téléphone, Internet \\
\hline Organisationnels & En développement soutenu & $\begin{array}{l}\text { Presse, radio, TV, firmes d'information } \\
\text { financière, cabinets comptables, firmes } \\
\text { de notation }\end{array}$ \\
\hline Institutionnels & Opérationnels à partir de 1934 & SEC, GAAP, blue sky laws (1910-20) \\
\hline \multicolumn{2}{|c|}{ Tendance générale : transferts massifs d'information de type 3 grâce aux janus } \\
institutionnels (années 1930)
\end{tabular}

\subsection{Le cas Enron et l'asymétrie d'information à l'époque de l'Internet}

59 Il est indispensable de consacrer une brève analyse à la situation actuelle marquée d'une part par la hausse spéculative de la fin des années 1990 suivie d'un effondrement aux allures de krach et, d'autre part, par une série inquiétante de scandales financiers qui indignent le public et qui incitent les autorités à y porter remède.

De notre point de vue, l'avènement de l'Internet pouvait signifier la fin pure et simple de l'asymétrie d'information. Ce janus technologique est si puissant qu'il met instantanément en contact tous les acteurs du marché, qu'ils soient initiés ou non, et il autorise toutes les configurations de communication imaginables. Non seulement l'information y circule librement, mais la Toile permet aux spéculateurs de contourner Wall Street en opérant avec des courtiers en ligne. Ainsi décrit, l'environnement financier des pays développés de la fin des années 1990 semblait avoir totalement réalisé l'hypothèse des marchés efficients où des millions d'individus rationnels et parfaitement bien informés évaluent instantanément et correctement le rendement de leurs investissements. Avec l'Internet, la main invisible du marché chère à Adam Smith avait apparemment trouvé l'instrument technique quasi-parfait de son avènement. Wall Street et Main Street paraissaient presque à égalité et sous de nombreux rapports, certains privilèges traditionnels de Wall Street semblaient même menacés (BusinessWeek 15 mars 1999).

61 Or, cette période de progrès apparemment décisifs se solde par un douloureux paradoxe. Des insiders ont alimenté le marché d'informations frauduleuses, les comptes de sociétés géantes ont été manipulés, des cabinets comptables ont menti sur les résultats d'entreprises pour préserver leurs activités de conseil. Comme sortant d'un rêve éveillé, chacun peut mesurer à quel point l'asymétrie d'information fait des ravages malgré Internet. Plus grave encore, chaque nouvelle révélation amplifie notre perception de l'asymétrie d'information au point qu'il est légitime de se demander aujourd'hui si Internet ne l'a pas aggravée au lieu de la résorber.

62 En bref, l'analyse montre que les informations de types 1, 2 et 3 qui doivent normalement se compléter pour informer le marché de façon efficace n'ont pas permis d'éviter une spéculation excessive et de signaler les fraudes. La régression est troublante, car la situation actuelle ressemble à de nombreux égards à celle qui prévalait avant les lois de $1934^{6}$. Elle évoque même les malversations qui avaient cours au XIX ${ }^{e}$ siècle, époque où les rares éléments-janus de transfert d'information n'en étaient qu'au stade embryonnaire. ${ }^{7}$. 
Comme dans les années trente Main Street se sent flouée par Wall Street et en appelle à la SEC pour assurer un transfert d'information équitable.

Comment en est-on arrivé là ? Ce type de situation complexe est évidemment le résultat de causalités multiples. L'accent est essentiellement mis sur l'analyse du problème en termes de transfert d'information.

De ce point de vue, l'origine de la dérive se trouve probablement dans le développement des stock options proposées aux dirigeants à partir de la fin des années 1980. Outre les problèmes comptables et fiscaux que posent les stock options, elles entraînent plusieurs effets pervers dans le domaine de l'information. En particulier, elles placent de fait les dirigeants en position d'insiders spéculatifs puisqu'ils ont intérêt à ce que les titres qu'ils peuvent acheter à bas prix grâce à leurs options gagnent rapidement en valeur boursière. De ce fait, ils sont incités à alimenter le marché en informations spéculatives (de type 1) alors que leur mission est de fournir aux parties prenantes (stakeholders) de l'entreprise des informations de type 3 (investissement à long terme). Des sociétés comme Enron ont donc maximisé les bonnes nouvelles (les bénéfices) et ont enterré les mauvaises (les dettes) dans les innombrables filiales créées à cet effet hors bilan (off balance sheet). Elles ont également manipulé les informations quand celles-ci n'étaient pas suffisamment positives. Enfin, elles ont été amenées à faire pression sur les cabinets d'audit pour qu'ils certifient de l'information comme étant de type 3 (investissement à long terme) alors qu'elle était non seulement de type 1 (spéculative) mais frauduleuse. Ce faisant, les cabinets d'audit responsables ont totalement failli à leur mission qui est de corriger l'asymétrie d'information au bénéfice des actionnaires et du grand public. Tout au contraire, ils ont œuvré en réalité pour un petit nombre d'insiders qui prenaient des positions spéculatives en bourse (Fusaro and Ross 2002 : 73, 127-128). L'analyse en termes de transfert d'information démontre que la crise de confiance de Main Street vis-à-vis de Wall Street provient en grande partie d'un mélange des genres dans le type d'information transférée.

Pour aggraver la situation, la SEC qui garantit le respect des règles du jeu ainsi que la qualité des éléments-janus mis au service du public n'a pas été en mesure de jouer convenablement son rôle. Dans un livre récent (Levitt 2002), le précédent président de la SEC, Arthur Levitt, explique la vanité de ses efforts quand il a tenté de dénouer le conflit d'intérêt inhérent à la double fonction des grands cabinets comptables. Ceux-ci interviennent fréquemment dans les mêmes entreprises en tant que consultant et en tant qu'auditeur. Ils s'y trouvent juge et partie, et leur activité de conseil est trop rémunératrice pour ne pas détériorer la qualité de leurs contrôles. Malgré ses efforts, Levitt n'a pu faire voter les lois nécessaires par le Congrès. L'enquête a montré que les réticences des sénateurs et des représentants avaient pour origine les dons généreux qu'ils avaient reçus de la part des Big Five, d'Enron et de leurs collègues (Fusaro and Ross 2002 : 139). Il a donc fallu qu'une crise éclate, que la bourse s'effondre et que les scandales se multiplient pour que la loi Sarbanes-Oxley soit votée en juillet 2002. Celle-ci tente de corriger les excès enregistrés dans les années 1990 et prévoit notamment la création d'un conseil de surveillance de la comptabilité (Public Company Accounting Oversight Board) qui semble concrétiser la proposition faite 45 ans plus tôt par Leonard Spacek, le successeur d'Arthur Andersen à la tête du cabinet du même nom. Spacek avait en effet milité pour la création d'une cour de la comptabilité (Accounting Court) qui superviserait les activités d'audit et de comptabilité aux États-Unis (Arthur Andersen \& Co 1963 : 122-123). Ce nouveau conseil apparait évidemment comme un janus institutionnel supplémentaire 
consacré à l'information de type 3, et notamment à l'information comptable qui s'est montrée très déficiente depuis une dizaine d'années. Sa création est probablement de nature à assurer un meilleur transfert de ce type d'information et de réduire l'asymétrie entre Wall Street et le grand public. Cela faisait sept ans que le sénateur Sarbanes et le représentant Oxley attendaient pour que leur loi soit votée par le Congrès. L'avenir nous dira si elle permettra de corriger efficacement l'asymétrie d'information qui a atteint ces dernières années des déséquilibres dignes de l'époque des Robber Barons du XIX ${ }^{\mathrm{e}}$ siècle.

\section{Conclusion}

Le modèle explicatif montre que la lutte contre l'asymétrie d'information a vigoureusement façonné l'évolution des bourses américaines depuis deux cents ans. Au cours de trois périodes historiques de durées approximativement équivalentes (60-70 ans environ) des types d'information financière de plus en plus sophistiqués se sont progressivement imposés pour assurer des transferts plus fiables et plus équitables entre les initiés et les non-initiés.

67 Après une période quasi exclusivement spéculative où les initiés détenaient le monopole de l'information, des janus technologiques et organisationnels ont assuré des transferts d'information continus et fiables qui ont contribué à construire une opinion publique boursière plus stable. Enfin, les janus institutionnels mis en place dans les années 1930 ont efficacement et durablement réduit l'asymétrie d'information même s'ils ne l'ont pas fait disparaître.

Ce travail peut se conclure par deux mises en perspective.

Le processus d'évolution présenté ici semble obéir à un schéma de développement de type tocquevillien (Tocqueville 1835 : 57-71). Il révèle en fait une démocratisation progressive de l'information boursière conduite par paliers successifs. Le monopole quasi absolu que les initiés de Wall Street exerçaient sur l'information au XIX siècle ressemble étrangement au pouvoir absolu dont les grands féodaux du Moyen-Age ou les princes de la Renaissance jouissaient en Europe. Les hommes d'affaires avisés que furent Henry Poor, John Moody, Charles Dow, Arthur Andersen, Charles Merrill, apparaissent comme des militants de cette démocratisation. Ils étaient en effet convaincus que transférer de l'information de qualité au grand public ne pouvait qu'être bénéfique à tous et s'inscrivait dans la marche du progrès économique. Les janus institutionnels comme la SEC sont au service du plus grand nombre et représentent la consécration politique de cette démocratisation finalement parachevée grâce à Internet.

Cependant, la crise actuelle qui est directement liée à une recrudescence de l'asymétrie d'information entre Wall Street et Main Street semble remettre en question ce processus démocratique. Si des scandales comme celui d'Enron, de WorldCom et d'Andersen ont pu se produire, l'asymétrie d'information s'est-elle réellement réduite ? Il est trop tôt pour répondre définitivement à cette question mais la suggestion suivante peut être avancée. Il a été observé que les paliers évolutifs marquant un progrès dans les transferts d'information se succédaient avec une certaine régularité, tous les 60-70 ans environ. C'est à peu près la durée qui sépare les années 1990 des lois des années 1930 (Gordon 1999: 294) et la crise actuelle indique peut-être que s'ouvre une nouvelle période marquée par de nouveaux éléments-janus. C'est en tout cas ce que semblent indiquer les 
lois récentes adoptées par le Congrès qui ne donnent peut-être qu'un avant-goût de réformes de plus grande ampleur à venir.

\section{BIBLIOGRAPHIE}

Angel, James J. 1998. Consolidation in the Global Equity Market: A Historical Perspective. <http:// msb.georgetown.edu/faculty/angelj/International_Competition2.pdf>.

Alvarez, Richard I. and Mark J. Astarita. 1995-2001. Introduction to the Blue Sky Laws. U.S. Securities and Exchange Commission. <www.seclaw.com/bluesky.htm>.

Arthur Andersen \& Co. 1963. The First Fifty Years. Chicago : Garland.

Business Week. 15 mars 1999. " Why the bourses are spooked; electronic rivals gain on the big board and Nasdaq ».

Chandler, Alfred D. Jr. 1981. Henry Varnum Poor, Business Editor, Analyst, Reformer. New York : Arno Press.

Colbert, David. 2001. Eyewitness to Wall Street: Four Hundred Years of Dreamers, Schemers, Busts and Booms. New York : Broadway Books.

Davis, James E. 1999. Corporate Disclosure Through the Stock Exchanges. Harvard Law School Third Year Paper. <cyber.law.harvard.edu/rfi/papers/disclose\%20abstract.htm>.

Frazer, James G. 1922 (première publication). The Golden Bough: A Study in Magic and Religion. Londres : Macmillan.

Fusaro, Peter C. and Ross M. Miller. 2002. What Went Wrong at Enron. New Jersey : John Wiley \& Sons.

Garvy, George. 1944. « Rivals and interlopers in the history of the New York security market ». The Journal of Political Economy. June 1944. L II, 128-143.

Geisst, Charles R. 1997. Wall Street, A History. New York : Oxford University Press.

Gordon, John Steele. 1999. The Great Game: A History of Wall Street. Londres : Orion Business Books.

Grimal, Pierre. 1999 [1945 Tome IV des Lettres de l'humanité. Les Belles Lettres. Paris]. Le dieu Janus et les origines de Rome. Paris : Berg International Éditeurs.

Hamilton, William Peter. 1989 [1922 New York : Harper \& Brothers]. The Stock Market Barometer. Detroit : Omnigraphics.

Keynes, John Maynard. 1936. General Theory. Londres : Macmillan.

Kindleberger, Charles P. 2002. Manias, Panics and Crashes, A History of Financial Crises. Basingstoke :Palgrave.

Levitt, Arthur. 2002. Take on the Street. New York : Pantheon/Random House.

Medbery, James K. 1968. [1870 Boston : Fields, Osgood \& Co]. Men and Mysteries of Wall Street. Fraser Publishing Company. Vermont : Wells.

Michie, R. C. 1987. The London and New York Stock Exchanges 1850-1914. Londres : Allen \& Unwin. 
Mishkin, Frederic S. 1991. « Asymmetric information and financial crises: A historical perspective ». In Hubbard, R. Glenn (ed.), Financial Markets and Financial Crises. Chicago : University of Chicago Press.

Moody, John. 1919. The Railroad Builders: A Chronicle of the Welding of the States. < www.blackmask.com/books14c/rroad.htm>.

New York Stock Exchange. Significant Historical Dates. <www.nyse.com/pdfs/historical99.pdf>.

Nobel Prize Lectures. 2001. Markets with Asymmetric Information. October 10, 2001. <nobel.se/ economics/laureates/2001/ecoadv.pdf>.

North, Douglass C. 1994. Institutions Matter. Economic History / Economics Working Paper Archive at WUSTL (Washington University). <http://netec.mcc.ac.uk/wopec/data/papers/ wpawuwpe9412001.html>.

Oesterle, Dale Arthur. 2000. « Securities market regulation: Time to move to a market-based approach ». Policy Analysis 374, June 21, 2000.

Pecora, Ferdinand. 1939. Wall Street Under Oath. New York : Simon and Schuster.

Perkins, Edwin J. 1999. Wall Street to Main Street: Charles Merrill and Middle-Class Investors. Cambridge : Cambridge University Press.

Philadelphia Stock Exchange. The History of the Philadelphia Stock Exchange. <www.phlx.com>.

Preda, Alex. 2002. On Ticks and Tapes: Financial Knowledge, Communicative Practices and Information Technologies on 19th Century Financial Markets. Paper prepared for the Columbia Workshop on Social Studies of Finance. May 3-5, 2002. <www.coi.columbia.edu/ssf/paper/preda.doc>.

Sobel, Robert. 1970. The Curbstone Brokers: The Origins of the American Stock Exchange. Washington D.C. : Beard Books.

Tocqueville, Alexis de. 1983 [1835]. De la démocratie en Amérique. Tome 1. Paris : Garnier Flammarion.

Wachtel, Howard M. 1996. Alexander Hamilton and the Origins of Wall Street. <http:// econwpa.wustl.edu:8089/eps/eh/papers/9610/9610001.html>.

\section{NOTES}

1. Mishkin propose une interprétation de l'histoire des crises financières du point de vue de l'asymétrie d'information. L'approche est économétrique et n'adopte pas la perspective de cet article, c'est-à-dire une caractérisation sociale et institutionnelle de l'asymétrie d'information (Mishkin 1991). Par ailleurs, James J. Angel utilise à plusieurs reprises la notion d'asymétrie d'information dans une perspective historique (Angel 1998).

2. Il épouse notamment sa démarche qui consiste à exposer son modèle explicatif (chapitre 2) et à en appliquer les différents éléments aux phases successives des crises financières (chapitres 4 à 9) (Kindleberger 2002).

3. La distinction est ancienne. Déjà en 1870, Medbury consacre le chapitre 9 de son ouvrage aux Great Operators (les insiders les plus importants de Wall Street) et le chapitre 10 aux Outsiders (Medbery 1870).

4. Gordon date des années 1830 le début de l'utilisation de l'expression Wall Street pour signifier l'ensemble du système financier américain (Gordon 1999 : 69). 
5. La plupart des historiens de Wall Street consacrent des descriptions pittoresques aux pratiques de cette période. Pour une présentation vécue et d'époque voir Medbury 1870 : chapitres 4 et 6 .

6. Ferdinand Pecora évoque le cas de la National City Company qui, en 1927, commit des malversations étonnamment similaires à celles d'Enron (Pecora 1939 : 119-121).

7. L'histoire des compagnies de chemin de fer américaines au XIX ${ }^{\mathrm{e}}$ siècle présente de nombreux parallèles avec le passage éclair d'Enron de la gloire à la banqueroute. Voir notamment le cas de la Baltimore \& Ohio (Moody 1919 : chapitre 5).

\section{RÉSUMÉS}

Cet article porte sur l'histoire boursière américaine et sur l'évolution de Wall Street. Il se propose de montrer à l'aide de la problématique de l'asymétrie d'information que les transferts d'information constituent depuis deux siècles l'un des moteurs les plus importants de l'évolution de Wall Street. Un modèle théorique d'explication est d'abord présenté. Il précise ce qu'on peut entendre par asymétrie d'information dans le contexte boursier et il dégage les notions d'initié ( insider) et de non-initié (outsider) ainsi que leurs extensions sociales, "Wall Street " et "Main Street». Il met ensuite en place les outils conceptuels de l'analyse : typologies des informations boursières et des moyens d'information appelés ici "éléments-janus ». Le modèle théorique propose enfin une grille de lecture applicable aux différentes périodes de l'histoire boursière américaine. Dans un second temps, le modèle explicatif est appliqué à trois périodes de cette histoire. Pour chaque période, la lecture adoptée permet de mettre en valeur des événements et des personnages que les interprétations historiques traditionnelles ignorent ou négligent. L'étude se conclut par une esquisse d'explication des récents scandales financiers du type Enron à partir de l'asymétrie d'information. La conclusion propose deux perspectives d'interprétation du sens de l'histoire boursière ainsi dégagé.

This article deals with the history of the U.S. securities markets and the evolution of 'Wall Street.' With the help of the concept of 'asymmetric information' the author intends to show that information transfers have been a major influence on the evolution of Wall Street for more than two centuries. First, an explanatory theoretical model is proposed. It defines the concept of 'asymmetric information' in the context of the securities markets. It clarifies the notions of 'insider' and 'outsider' and their social development in the U.S. ('Wall Street' and 'Main Street'). Then, a conceptual framework is proposed. It includes the typologies of stock market information and of information transfers (called 'janus-elements' in the framework). Using this model, the different periods in the history of the U.S. securities markets can be analyzed and interpreted. Second, the model is applied to three periods in the history of the securities markets. This study will highlight people and events which traditional historical interpretations tend to overlook or ignore. The presentation will then try to throw some light on the Enron-type scandals from the viewpoint of information asymmetrics and information transfer. Finally, the conclusion draws two interpretations from the analysis. 
INDEX

Mots-clés : asymétrie d'information, bourse américaine, histoire financière, Main Street, New York Stock Exchange, Wall Street

Keywords : asymmetric information, financial history, U.S. stock market

\section{AUTEUR}

\section{MICHEL VAN DER YEUGHT}

Michel Van der Yeught est maître de conférences au département GEA de l'IUT de Toulon. Il enseigne l'anglais de la finance et de la comptabilité et s'est spécialisé dans la culture boursière anglo-saxonne.mvdy@free.fr 